\title{
Acquiring complex partial orderings in comparison with acquiring similar-sized linear orderings
}

\author{
SHANNON DAWN MOESER \\ Memorial University of Newfoundland, St. John's, Newfoundland AlB 3X8, Canada
}

\begin{abstract}
The acquisition of a 14-term partial ordering was compared with the acquisition of a 14-term linear ordering. Learning the partial ordering was found to be more difficult because of two factors: (1) Subjects do not appear to have in their knowledge systems a prototype or rule for representing a long list of comparative relations as a partial ordering structure, and (2) the partial ordering must be presented so that some of the adjacently presented premises do not contain a common element. When these two factors were controlled, the partial ordering was as easy to learn as the linear ordering. It was also found that subjects learning the partial ordering showed no evidence of a serial position learning curve, whereas subjects learning the linear ordering showed some tendency to produce a serial position learning curve.
\end{abstract}

There have been many investigations of how linear order information is encoded and retrieved. In these studies, subjects are presented a set of propositions that describe a linear ordering of comparative relations. An example of such a set is "Mary is older than Judy, Judy is older than Anne, and Anne is older than Lynn." The information in these sentences can be combined to form a connected chain of premises; namely, "Mary is older than Judy who is older than Anne who is older than Lynn." Most investigations of linear orderings have concluded that subjects encode the information from this type of input into a single integrated construct. One line of evidence used to support this conclusion is the finding of a serial position learning curve. In learning a set of individual comparisons, subjects make more errors on the pairs in the middle of the ordering than on those at the ends (e.g., Riley \& Trabasso, 1974; Trabasso, Riley, \& Wilson, 1975). Bower (1971) has argued that such effects are prima facie evidence of an integrated construction.

However, the serial position learning curve has not been found with partial orderings of comparative relations. Like linear orderings, partial orderings involve a sequence of linked items that form a connected chain of premises. Unlike linear orderings, the chain involves branches. For example, compare the topographical properties of the simple linear ordering illustrated in Figure 1a with the partial ordering shown in Figure $1 \mathrm{~b}$. Both orderings contain 14 linked items and can be described by a set of 13 comparative premises. In the

This research was supported by Grant A9638 from the National Research Council of Canada. Reprint requests should be sent to Shannon Moeser, Institute for Research in Human Abilities, Memorial University of Newfoundland, St. John's, Newfoundland A1B 3X8, Canada.

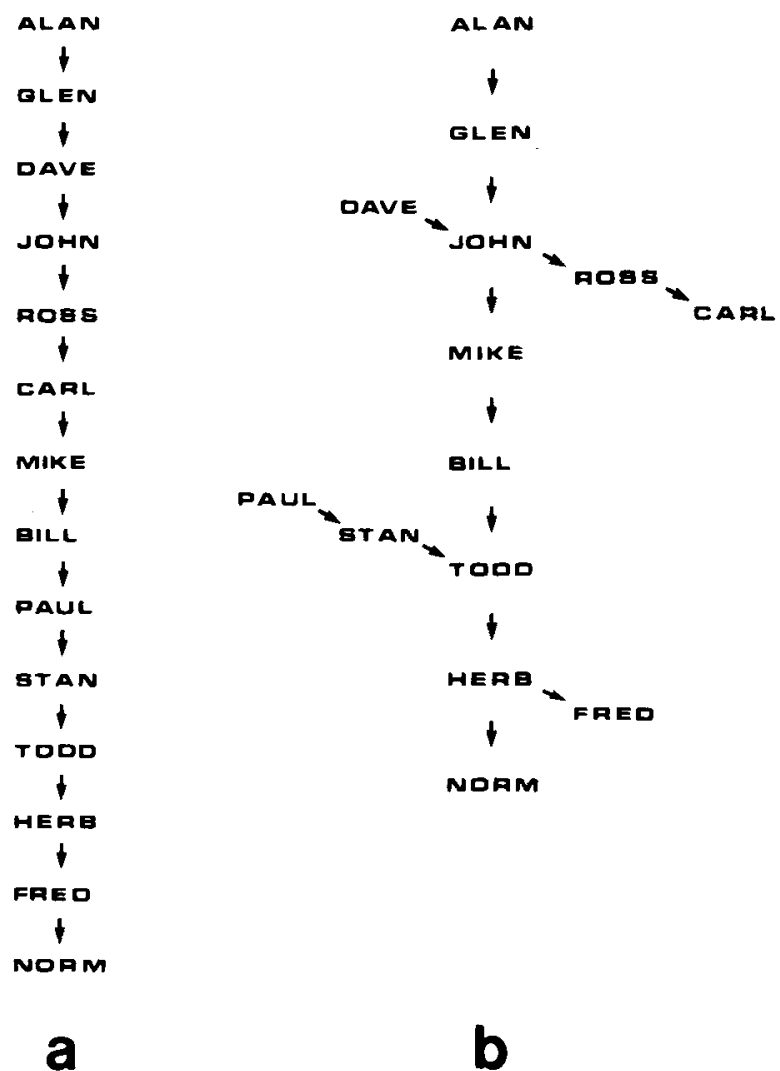

Figure 1.

linear ordering, however, an item can have at most two direct connections to other items, one "greater than" connection and one "less than" connection. Also, in a linear ordering there will always be two items that have only one direct connection to other items in the chain: the two ends of the chain. In the 
partial ordering, a given item might have several direct connections to other items. For instance, in Figure 1b, the item "John" has two "greater than" direct connections and two "less than" direct connections. Also, in the partial ordering there are more than two end items; in Figure 1b, "Alan," "Dave," "Carl," "Paul," "Fred," and "Norm" are all ends of a chain, that is, items with only one direct connection to another item.

There have been very few experiments using partial orderings. Hayes-Roth and Hayes-Roth (1975) used a partial ordering to investigate differences in amount of time required to verify various comparative relationships. They found that in contrast to the results reported on linear ordering sets, subjects took much more time to verify nonadjacent relations they had not learned than to verify the adjacent relations they had learned. On the basis of this evidence, Hayes-Roth and Hayes-Roth argued that a partial ordering was processed in a qualitatively different way from a linear ordering.

In answer to Hayes-Roth and Hayes-Roth (1975), Moeser and Tarrant (1977) argued that subjects can represent a set of comparisons in two ways, either as a holistic unit or as a set of independent episodes. They suggested that the discrepency between the Hayes-Roth and Hayes-Roth results and those obtained using linear orderings was due to the fact that Hayes-Roth and Hayes-Roth presented their comparisons in a nonsequential order, that is, so that no two comparisons containing the same name occurred sequentially. This is a presentation format that generally encourages subjects to store related items independently of each other (cf. Moeser, 1977). In most linear ordering experiments, the items are presented in a logical sequence, either from the greatest to the least or from the least to the greatest. This type of presentation method generally encourages subjects to store related items as a single unit of information (e.g., Moeser, 1977, 1978). When Moeser and Tarrant (1977) presented the comparisons from a partial ordering in such a way as to encourage their storage as a single unit, they found no difference in reaction times between adjacent and nonadjacent comparisons. Thus, their results did not duplicate the findings of HayesRoth and Hayes-Roth (1975). However, the Moeser and Tarrant study did not duplicate the findings of reported linear ordering studies either, for they found no evidence of a serial position learning curve. In fact, in the sequential presentation condition, subjects appeared to employ a "sequential" learning strategy. That is, the items at the beginning of the sequence were learned first, and those at the end of the sequence were learned last. Nevertheless, Moeser and Tarrant argued that the fact that their subjects were able to answer the nonadjacent questions as easily as the adjacent questions demonstrated that the partial ordering was represented as a holistic unit in this study, and so the serial position lorrning curve might be a specific effect obtained with linear ordering structures.
The Moeser and Tarrant (1977) study was specifically designed to show that Hayes-Roth and Hayes-Roth (1975) had used an inappropriate technique in their investigations of a partial ordering. In order to duplicate the Hayes-Roth and Hayes-Roth procedure, the subjects received eight presentations of the set of comparisons before ever being tested on their ability to remember these comparisons. Consequently, it was not a well controlled study of acquisition processes in partial orderings. After the eight presentations, several subjects had perfect scores on the memory test, and so the acquisition data described were those obtained from the poorer subjects in the experiment. The experiments in the present paper were designed to observe the acquisition process in a more systematic fashion.

Comparisons of partial orderings with linear orderings have not received much experimental interest. DeSoto and his colleagues (DeSoto, 1960, 1961; DeSoto, London, \& Handel, 1965) have suggested that partial orderings are more difficult to construct than linear orderings of similar length. However, a recent study by Smith and Mynatt (Note 1) found partial orderings to be no more difficult to learn than linear orderings. In the Smith and Mynatt study, four- and five-item arrays were used; with such small partial orderings, the pairs must be presented in a sequential order. That is, each pair must contain an element in common with the pair presented immediately before it (e.g., $A>B, B>C$, B $>$ D). With more complex partial orderings, such as the one used by Moeser and Tarrant (1977), it is impossible to use a sequential order. No matter how the items are presented, there must be some adjacently presented pairs that do not contain a common element. Foos, Smith, Sabol, and Mynatt (1976) have demonstrated that it is more difficult to construct an integrated array from a nonsequential or "nonmatch" presentation order than from a sequential or "match" presentation order. Thus, the difficulty in constructing complex partial orderings may arise from the difficulty found in constructing any arrays containing a nonmatch component. The present experiments were designed to provide information on this question as well.

\section{EXPERIMENT 1}

\section{Method}

Materials. Two sets of comparative orderings were constructed. Each contained 14 elements denoted by male names and each "greater than" link between elements was represented by the relation "older than." One of the structures was a linear ordering constructed from 13 comparative premises that could be arranged in a sequential series from oldest to youngest. The other structure was a partial ordering, also constructed from 13 comparative premises. The main axis of the partial ordering consisted of seven comparative pairs. In addition, there were three side branches grafted onto the main axis; these consisted of three premises, two premises, and one premise, respectively. A topological illustration of these two structures is shown in Figure 1.

The comparisons were presented in the form of statements 
about age relations (e.g., Alan is older than Glen). In the partial ordering condition, the seven pairs on the main vertical axis were presented first using a sequential order (e.g., Alan $>$ Glen, Glen $>$ John, John $>$ Mike, Mike $>$ Bill, Bill $>$ Todd, Todd $>$ Herb, and Herb $>$ Norm), then the three branching pairs near the top of the axis (e.g., Dave $>$ John, John $>$ Ross, and Ross $>$ Carl), the two branching pairs further down the axis (e.g., Paul $>$ Stan and Stan $>$ Todd), and finally, the pair on the bottom branch (eg., Herb $>$ Fred). The presentation order in the linear ordering condition was designed to be as similar to this as possible. The first seven pairs on the axis were presented in sequential order (e.g., Alan $>$ Glen, Glen $>$ Dave, Dave $>$ John, John $>$ Ross, Ross $>$ Carl, Carl $>$ Mike, and Mike $>$ Bill). This was followed by three pairs from near the bottom of the axis (e.g., Stan $>$ Todd, Todd $>$ Herb, and Herb $>$ Fred), then two pairs connecting the two presented segments (e.g., Bill $>$ Paul and Paul $>$ Stan), and, finally, the bottom pair on the array (e.g., Fred $>$ Norm). Although these two presentation orders are not identical, both involve four groups of sequential sets-one containing seven pairs, one containing three pairs, one containing two pairs, and one containing one pair-and both involve three nonmatched adjacent pairs forming the transitions between the four sequential groups.

As can be seen in Figure 1, the names in the two types of orderings are matched as closely as possible. For example, in both, Alan, Glen, Dave, and John are at the top of the chart and Todd, Herb, Fred, and Norm are at the bottom. Five such matched structures were created, using the same 14 names shown in Figure 1, but each pair of structures randomly varied the positions of these names.

Two kinds of relations exist in the orderings: (1) those between adjacent elements (e.g., Mike $>$ Bill) and (2) those between nonadjacent elements (e.g., John $>$ Bill). In the linear ordering, John and Bill are connected indirectly by four links; in the partial ordering, John and Bill are connected indirectly by two links. As only adjacent comparisons were presented to the subjects, the relationships between nonadjacent elements would have to be inferred by using the connections via the transitive operator "older than."

For each ordering condition, a recognition test was created. This test consisted of 26 two-alternative questions. One alternative was a true premise, and the other was the false reversal of the true premise. There were two versions of the recognition test, one given to the subjects receiving the linear ordering and the other to the subjects receiving the partial ordering. Both versions contained the 13 adjacent comparisons and their false

Table 1

Nonadjacent Comparisons Used in the Recognition Test in Experiment 1, Using the Name Relations in Accordance with the Mustrations Shown in Figure 1

\begin{tabular}{ll}
\hline Linear Ordering & \multicolumn{2}{c}{ Partial Ordering } \\
\hline 1. Glen $>$ John & 1. Paul $>$ Todd \\
2. Mike $>$ Paul & 2. Dave $>$ Ross \\
3. Ross $>$ Mike & 3. Bill $>$ Herb \\
4. Todd $>$ Fred & 4. Glen $>$ Bill \\
5. Bill $>$ Stan & 5. John $>$ Carl \\
6. Stan $>$ Herb & 6. John $>$ Bill \\
7. Paul $>$ Todd & 7. Stan $>$ Herb \\
8. Dave $>$ Ross & 8. Dave $>$ Mike \\
9. Carl $>$ Bill & 9. Glen $>$ Ross \\
10. Paul $>$ Herb & 10. Mike $>$ Todd \\
11. Mike $>$ Stan & 11. Mike $>$ Herb \\
12. John $>$ Mike & 12. John $>$ Todd \\
13. John $>$ Bill & 13. John $>$ Herb \\
\hline
\end{tabular}

reversals, and both contained 13 nonadjacent comparisons and their false reversals. Table 1 lists the nonadjacent questions used in the recognition test in reference to the illustrations shown in Figure 1. As can be seen, in both encoding conditions, the 13 nonadjacent comparison questions consisted of nine items with two links between elements, three items with three links between elements, and one item with four links between elements. In the linear condition, all of the nonadjacent questions compare items lying along a single axis. In the partial ordering conditions, 3 of the 13 nonadjacent questions compared items lying along two axes (Stan $>$ Herb, Dave $>$ Mike, and Glen $>$ Ross). In order to answer these three questions subjects would have to correctly represent the intersections between the branches of the partial ordering. The same recognition test was used for each of the five different name structures, with appropriate changes made in accordance with the way in which the names were structured in relation to each other.

Subjects. Ten male and 10 female subjects were assigned to each condition. These were first-year undergraduates enrolled at Memorial University of Newfoundland. They were tested in groups of four, and each group received a different random ordering of names. The subjects were paid $\$ 3$ for participating in the 1-h experiment and were given a bonus of 50 cents for each perfect score obtained on any test.

Procedure. The subjects were told that they would see a set of statements and would be tested on their ability to remember these statements. They were told that they would receive four similar trials, and that they could earn an extra 50 cents for each test on which they obtained a perfect score.

The experiment consisted of four phases. First was the acquisition phase, wherein the 13 true adjacent comparisons were presented on $35-\mathrm{mm}$ slides by a Kodak $850 \mathrm{H}$ slide projector. Each slide remained on the screen for $5.0 \mathrm{sec}$, with an interslide interval of $.8 \mathrm{sec}$. The acquisition phase was followed by a 2 -min distractor task consisting of a set of orally presented arithmetic problems. The distractor task constituted the second phase of the experiment. The third phase consisted of the recognition test, which was also presented by the slide projector. Each test slide remained on the screen for $8.0 \mathrm{sec}$, with an interslide interval of $.8 \mathrm{sec}$. To answer the recognition test questions, subjects had to circle either "a" or "b" on an answer sheet. The fourth phase was an illustration test. Immediately following the recognition test, the subjects were given $3 \mathrm{~min}$ to draw an illustration of the relationships they had learned. These four phases were repeated four times. On each trial the recognition test had identical questions, but these questions were presented in a different order. A different set of arithmetic problems was used each time.

\section{Results}

Recognition test. Each subject received a total correct score on the recognition test for each trial. Each group's mean proportion of correct scores on this test is shown in Table 2. As can be seen, the scores for the linear ordering group were higher than those for the partial ordering group. A group by trial analysis of variance on the individual scores showed a significant difference between groups $[\mathrm{F}(1,38)=9.60, \mathrm{p}<.01$, $\mathrm{MSe}=24.50]$ and a significant difference among trials $[\mathrm{F}(3,114)=48.76, \mathrm{p}<.001, \mathrm{MSe}=2.72]$. The interaction between groups and trials was not significant $[\mathrm{F}(3,114)=.62, \mathrm{p}>.10, \mathrm{MSe}=2.72]$. The difference between groups reflects the fact that the linear ordering group performed better on the recognition test than the 
Table 2

Mean Proportion of Correct Scores on the Recognition Test and the Illustration Test for the Two Experimental Conditions in Experiment 1

\begin{tabular}{cccccc}
\hline & \multicolumn{2}{c}{ Recognition Test } & & \multicolumn{2}{c}{ Illustration Test } \\
Trial & $\begin{array}{c}\text { Partial } \\
\text { Ordering }\end{array}$ & $\begin{array}{c}\text { Linear } \\
\text { Ordering }\end{array}$ & $\begin{array}{c}\text { Partial } \\
\text { Ordering }\end{array}$ & $\begin{array}{c}\text { Linear } \\
\text { Ordering }\end{array}$ \\
\hline 1 & .52 & .59 & .15 & .18 \\
2 & .58 & .68 & .25 & .31 \\
3 & .63 & .72 & .33 & .47 \\
4 & .67 & .78 & .38 & .60 \\
\hline
\end{tabular}

partial ordering group; the difference among trials reflects the fact that overall performance improved as the experiment proceeded.

The recognition test scores were also differentiated in terms of adjacent and nonadjacent questions. A group by question type analysis of variance found a significant difference between groups $[F(1,38)=9.60$, $\mathrm{p}<.01, \mathrm{MSe}=48.76$, but neither a significant difference between question types $[F(1,38)=.08, p>.10$, $\mathrm{MSe}=5.62$ ] nor a significant interaction between groups and question types $[\mathrm{F}(1,38)=.04, \mathrm{p}>.10$, $\mathrm{MSE}=5.62 \mathrm{]}$. This meant that in both groups the subjects were just as good at recognizing the correct adjacent relationships (which were presented during the acquisition phase) as they were at recognizing correct nonadjacent relationships (which had to be inferred from the information they were given). This finding differs from reports indicating that with linear orderings, at least, nonadjacent comparisons are more easily learned than adjacent comparisons (e.g., Potts, Banks, Kosslyn, Moyer, Riley, \& Smith, 1978).

Illustration test. To illustrate what they had learned, none of the subjects produced statements such as those they were shown during the acquisition phase of the experiment. Most drew a vertical or horizontal line of names and wrote "older" at the top of the line or on its left side and "younger" at the bottom of the line or on its right side. None of the subjects in the partial ordering condition formed any facsimile of a partial ordering; that is, they did not attempt to use branches. Two subjects in this condition drew two separate arrays, but all of the other subjects tried to integrate the information into a single linear array. These illustrations indicate that in both conditions, most subjects tried to arrange the comparative information into some sort of holistic array and did not attempt to memorize the adjacent comparisons as a set of independent premises.

The illustrations were scored by giving 1 point for each adjacent pair in which the two items were correctly related to each other. Thus, if a subject in the linear condition who received the ordering shown in Figure 1a produced a line showing Alan $>$ Dave $>$ John $>$ Carl $>$ Mike, 2 points would be given, 1 for the Dave $>$ John pair and 1 for the Carl $>$ Mike pair. This meant that in both ordering conditions, the highest score that could be obtained was 13. Each group's mean proportion of correct scores on the illustration test are also shown in Table 2. Again, the scores for the linear ordering group were higher than those for the partial ordering group. A group by trial analysis of variance on the individual scores showed a significant difference between groups $[F(1,38)=7.45, p<.01, \mathrm{MSe}=11.49]$, a significant difference among trials $[F(3,114)=97.65, p<.001$, $\mathrm{MSe}=1.43 \mathrm{]}$, and a significant interaction between groups and trials $[\mathrm{F}(3,114)=8.15, \mathrm{p}<.01, \mathrm{MSe}=1.43]$. Thus, as was found with the recognition test, the linear ordering group was significantly better than the partial ordering group, and as the trials progressed, the subjects in both groups improved in their performance. With the illustration test, however, subjects in the linear ordering group improved more rapidly as the experiment progressed than did subjects in the partial ordering group.

Perfect scores. The subjects were encouraged to learn the comparison information by the promise of 50 cents for each perfect score they obtained. None of the subjects obtained a perfect score on any recognition test; one subject in the linear ordering condition obtained a perfect score on the illustration test on the fourth trial.

\section{Discussion}

On the final test, performance in both experimental conditions was quite poor. This suggests that not enough trials were given to allow subjects to acquire a thorough knowledge of the complex arrays. Nevertheless, even with only four trials, it was obvious that subjects in the linear ordering condition were progressing at a faster rate than were subjects in the partial ordering condition. This occurred in spite of the fact that in both conditions, a "nonmatch" presentation format was used.

At least one reason for the difference in learning rates was suggested by the types of illustrations produced by subjects in the partial ordering condition. Subjects simply did not appear to have at their disposal a way of representing a partial ordering in a graphical form. Instead, most of them attempted to represent the comparative premises in the form of a linear array.

Moeser and Tarrant (1977) found that subjects who did not form a correct mental representation of a partial ordering performed better on the adjacent comparisons than on the nonadjacent comparisons. This finding was not replicated in Experiment 1. However, in the Moeser and Tarrant study, the adjacent comparisons were presented eight times in succession before any test was given; then subjects were trained on the adjacent comparisons until they reached a criterion on two consecutive correct trials. Only then were they given both adjacent and nonadjacent comparisons. In the present study, subjects saw only one presentation of the adjacent set before they were tested on both adjacent and nonadjacent comparisons. The illustrations suggest that 
within the constraints of their knowledge, the subjects were attempting to form holistic arrays. By forming holistic arrays, they were able to make judgments about adjacent and nonadjacent comparisons with approximately equal accuracy. For example, a subject asked to form an array like that shown in Figure $1 \mathrm{~b}$ might produce the following linear array: Alan $>$ Glen $>$ Dave $>$ John $>$ Ross $>$ Carl $>$ Bill $>$ Mike $>$ Stan $>$ Todd $>$ Fred. With this knowledge, the subject should answer the recognition test questions as follows. (1) With the adjacent comparison questions, correct answers should occur on eight comparisons: Alan $>$ Glen, Glen $>$ John, John $>$ Mike, Bill $>$ Todd, Dave $>$ John, John $>$ Ross, Ross $>$ Carl, and Stan $>$ Todd. An incorrect answer should occur on one comparison: Mike $>$ Bill. Guesses should occur on four comparisons: Paul $>$ Stan, Todd $>$ Herb, Herb $>$ Fred, and Herb $>$ Norm. (2) With the nonadjacent comparison questions, correct answers should occur on eight comparisons: John $>$ Bill, John $>$ Carl, John $>$ Todd, Mike $>$ Todd, Dave $>$ Ross, Glen $>$ Ross, Glen $>$ Bill, and Dave $>$ Mike. Guesses should occur on five comparisons: Stan $>$ Herb, John $>$ Herb, Bill $>$ Herb, Paul $>$ Todd, and Mike $>$ Herb .

This is, of course, only one example, but it demonstrates why there were probably no differences between adjacent and nonadjacent questions on the recognition test. In the Moeser and Tarrant (1977) experiment, on the other hand, the training progressed to the point at which the subjects simply learned the individual premises without placing them into a single array. When this occurred, performance on adjacent comparisons was better than performance on nonadjacent comparisons.

\section{EXPERIMENT 2}

In Experiment 1, subjects in the partial ordering condition were unable to produce a graphical representation of a partial array. As well, however, their performance on the recognition test indicated that they had failed to adequately learn the adjacent comparisons presented during the acquisition phase of the experiment. Therefore, it is possible that once the adjacent pairs were learned to a high criterion, the subjects would eventually discover a method whereby they could represent the partial ordering in such a way that they would be able to make correct judgments about all relationships in the array. This was the hypothesis tested in Experiment 2. Instead of receiving four trials, subjects in the partial ordering condition received eight trials. If they were capable of learning how to represent a partial ordering, at least some of them should demonstrate this by the end of the eighth trial.

\section{Method}

Materials and Procedure. In Experiment 2, subjects were tested only in a partial ordering condition. The same materials and procedure were used as were used in Experiment 1, except that eight trials were given instead of four. Also, in Experiment 2 , subjects were told whether they had received a perfect score on either of the tests at the end of each trial.

Subjects. Ten male and 10 female subjects were tested in the experiment. These were first-year undergraduates enrolled at Memorial University of Newfoundland. They were tested in groups of four, and each group received a different random ordering of names. The subjects were paid $\$ 6$ for participating in the 2-h experiment and were given a bonus of 50 cents for each perfect score obtained on any given test.

\section{Results}

Recognition test. On the recognition test, each subject received a total correct score for adjacent comparisons and a total correct score for nonadjacent comparisons for each trial. The mean proportions correct are shown in Table 3. A question type by trial analysis of variance on the individual scores showed a significant difference between question types $[F(1,19)=$ 23.47, $\mathrm{p}<.001, \mathrm{MSe}=3.78]$, a significant difference among trials $[F(7,133)=22.54, \mathrm{p}<.001, \mathrm{MSe}=2.09]$, and a significant interaction between question types and trials $[F(7,133)=8.47, \mathrm{p}<.01, \mathrm{MSe}=1.15]$. The difference between question types reflects the fact that the subjects performed better on the adjacent questions than on the nonadjacent questions; the difference among trials reflects the fact that overall performance improved as the experiment proceeded; the significant interaction reflects the fact that subjects progressed faster on the adjacent questions as the experiment proceeded than they did on the nonadjacent questions.

There were two types of nonadjacent questions. Ten of the 13 questions involved inferring relationships that lay along a single axis, whereas 3 of these questions involved inferring relationships that lay along two axes of the ordering. The subjects got only $49 \%$ of the latter three questions correct when their scores were summed across trials; in other words, they did no better than chance on the two-axis nonadjacent questions. On the other hand, the group got $66 \%$ of the 10 single-axis nonadjacent questions correct when their scores were summed across trials. Thus, subjects performed much better on the nonadjacent questions lying along a single axis than they did on the nonadjacent questions lying along two axes.

Table 3

Mean Proportion of Correct Scores on the Recognition Test and the Ilustration Test in Experiment 2

\begin{tabular}{cccc} 
& \multicolumn{3}{c}{ Recognition Test } \\
\cline { 2 - 3 } Trial & $\begin{array}{c}\text { Adjacent } \\
\text { Pairs }\end{array}$ & $\begin{array}{c}\text { Nonadjacent } \\
\text { Pairs }\end{array}$ & $\begin{array}{c}\text { Illustration } \\
\text { Test }\end{array}$ \\
\hline 1 & .50 & .50 & .15 \\
2 & .57 & .56 & .20 \\
3 & .63 & .61 & .29 \\
4 & .66 & .61 & .41 \\
5 & .76 & .63 & .57 \\
6 & .80 & .65 & .70 \\
7 & .85 & .68 & .82 \\
8 & .87 & .74 & .88 \\
\hline
\end{tabular}


Illustration test. The illustration test was scored as in Experiment 1, and the mean proportion of correct scores is also shown in Table 3. On this test, there was a significant difference among trials $[F(7,133)=139.94$, $\mathrm{p}<.001, \mathrm{MSe}=1.89]$, indicating that subjects improved in performance as the trials progressed. Although performance on the illustration test was very good at the end of the experiment, none of the subjects drew an illustration of a partial ordering. In other words, they had learned very well the adjacent premises presented during the acquisition phase, but they illustrated these incorrectly. On the last trial, eight of the subjects were still trying to form the comparisons into a single integrated linear array; four wrote down each of the 13 presented premises; and eight drew four separate linear arrays, one for each of the sections of the partial ordering. Because the illustration test was scored in terms of correct adjacent pairs, subjects could receive a perfect score on the illustration test even if the relationships among the branches of the array were not correctly represented. Thus, although 12 subjects received perfect illustration test scores on the last trial, none illustrated the information in such a way that they could answer nonadjacent questions lying along two branches of the array.

Perfect scores. On the illustration test, one subject received a perfect score on Trial 6 , five subjects received perfect scores on Trial 7 , and 12 subjects received perfect scores on Trial 8 . On the recognition test, no subject received a perfect score. However, on the adjacent comparison questions, there were six perfect scores in Trial 7 and seven in Trial 8 . There was only one perfect score obtained on the nonadjacent questions; this occurred on Trial 7.

\section{Discussion}

Although several subjects in Experiment 2 learned all of the adjacent pairs presented during the acquisition phase, none of them was able to form a correct visual illustration of a partial ordering. Furthermore, they did not appear to be able to form a correct mental representation either, as their performance on the nonadjacent questions in the recognition test was quite poor in the latter trials, whereas their performance on adjacent questions was quite good on the same trials. Several of the nonadjacent questions in the recognition test required that subjects possess a knowledge of the interrelations among different sections of the parital ordering, and performance was poorer on these questions than on those requiring judgments about relations in a single section of the array. Consequently, it appears that the subjects' failure to learn the partial ordering arose from their failure to represent this ordering as a set of intersecting branches.

In contrast to Experiment 1, in Experiment 2 subjects were better at answering the adjacent questions on the recognition test than at answering the nonadjacent questions. This difference occurred as the subjects acquired the information that was presented during acquisition. As learning progressed, there was a change in learning strategy for most subjects: At first, they attempted to integrate all information into a single linear array; later, many learned to form several independent linear arrays, even though these independent arrays contained some identical names. A few other subjects simply learned the individually presented premises. Consequently, although the subjects showed consistent improvement in learning the adjacent comparisons, they showed much slower progress in learning the nonadjacent comparisons. Thus, it appears that performance will be better on adjacent comparisons than on nonadjacent comparisons if two criteria are met: (1) The adjacent comparisons must be well learned, and (2) the subjects have not formed a correct mentai representation of the partial ordering.

\section{EXPERIMENT 3}

Experiments 1 and 2 demonstrated that subjects do not form a mental representation of a partial ordering when presented a set of adjacent comparisons. Instead, they tend to use this information to form a representation of a linear array. Moeser and Tarrant (1977) found, however, that subjects were quite capable of forming a representation of a partial ordering when instructed on their nature. Therefore, Experiment 3 was designed to test whether subjects are poorer at learning partial orderings only because they do not know what type of representation they are expected to form or whether, even with this knowledge, there is still something about the nature of partial orderings that makes them more difficult to learn than linear orderings.

In a pilot study, the author found that subjects instructed on how to form partial orderings were still poorer on performance tests than were subjects given a linear ordering containing the same number of comparative premises. However, the linear ordering used in the pilot study contained no nonmatch components. Thus, the superior performance in the linear ordering could have been due to the fact that the partial ordering did contain nonmatch components. Therefore, in Experiment 3 , there were three encoding conditions. There was a partial ordering condition and a linear nonmatch condition that were identical to those used in Experiment 1 . In addition, there was a linear match condition wherein all the premises were presented in sequential order. It is the latter type of presentation order that is generally used in experiments investigating the acquisition of linear orderings.

\section{Method}

Methods and Procedure. The materials and procedure of Experiment 3 were identical to those used in Experiment 1 with the following exceptions: (1) In addition to the partial ordering and linear nonmatch conditions used in Experiment 1, there was a linear match condition, wherein the 13 adjacent comparisons were presented in a sequential order during the acquisition 
phase of the experiment. In Figure 1a, a sequential order would be Alan $>$ Glen, Glen $>$ Dave, Dave $>$ John, John $>$ Ross, Ross $>$ Carl, Carl $>$ Mike, Mike $>$ Bill, Bill $>$ Paul, Paul $>$ Stan, Stan $>$ Todd, Todd $>$ Herb, Herb $>$ Fred, and Fred $>$ Norm. (2) At the beginning of the experimental session, the subjects received a written set of instructions that explained how to construct partial orderings, linear nonmatch orderings, or linear match orderings. (The particular instructions depended on the condition in which the subjects were being tested.) These instructions consisted of three examples of comparative relations that used the transitive elements "taller than" (with girls' names), "bigger than" (with dogs' names), and "smarter than" (with animals' names). The first example listed a set of eight adjacent comparisons, and accompanying this list was a topological illustration showing how the set of comparisons could be represented as a visual array. Beneath this illustration was a set of test questions, containing both adjacent and nonadjacent comparisons, that explained in detail why each question was true or false. The second example listed a set of 10 adjacent comparisons and requested that the subject draw a topological illustration of this set. This was followed by a set of test questions that contained both adjacent and nonadjacent comparisons, which the subject was required to answer. When the subjects completed the second example, the experimenter discussed these test questions with them. The third example consisted of a set of nine adjacent comparisons that were read slowly by the experimenter. While the experimenter was reading the comparisons, the subjects were required to draw a topological illustration representing the comparison information. Then the experimenter read a set of adjacent and nonadjacent comparison questions, and the subjects were required to write down whether each was a true statement or a false statement. In order to proceed through the rest of the experiment, the subject had to have a perfect score on the test questions of this third example and draw the illustration correctly. All subjects who participated in the experiment passed this criterion. (3) There were six trials given instead of four. (4) As in Experiment 2, subjects were told whether they had received a perfect score on either test at the end of each trial.

Subjects. Ten male and 10 female subjects were assigned to each condition. All were first-year undergraduates attending Memorial University of Newfoundland. They were tested in groups of four, and each group received a different random ordering of names. The subjects were paid $\$ 6$ for participating in the 2-h experiment and were given a bonus of 50 cents for each perfect score obtained on any test.

\section{Results}

Recognition test. On the recognition test, each subject received a total correct score on adjacent comparisons and a total correct score on nonadjacent comparisons for each trial. The mean proportions correct for each encoding condition are shown in Table 4 . A group by trial by question type analysis of variance on the individual scores showed a significant difference among groups $[\mathrm{F}(2,57)=15.65, \mathrm{p}<.01, \mathrm{MSe}=11.67]$ and a significant difference among trials $[F(5,285)=123.15$, $\mathrm{p}<.001, \mathrm{MSe}=2.87]$. There were no other significant effects. The significant difference among trials reflects the fact that performance improved as the experiment proceeded. As can be seen in Table 4, performance in the partial ordering and linear nonmatch groups was approximately equal. Therefore, the significant difference among groups probably was due to the fact that performance in the linear match group was considerably better than performance in the other two groups. The Newman-Keuls analysis comparing the three groups supports this suggestion. The linear match group was significantly better than both of the other two groups $(p<.01)$, but there was no significant difference between the partial ordering and the linear nonmatch groups.

Illustration test. The illustration test was scored as in Experiments 1 and 2. The mean proportion of correct scores obtained by the three encoding conditions is shown in Table 5. A group by trial analysis of variance on the individual scores found a significant difference among groups $[\mathrm{F}(2,57)=9.28, \mathrm{p}<.01, \mathrm{MSe}=21.81]$ and a significant difference among trials $[F(5,285)=$ $217.40, \mathrm{p}<.001, \mathrm{MSe}=3.30]$. Again, the significant difference among trials was caused by an overall improvement in performance as the experiment proceeded, and the significant difference among groups seemed due to the better performance shown by the linear match group. A Newman-Keuls analysis comparing the three groups found the linear match group significantly better than both of the other two groups $(p<.01)$ and no significant difference between the partial ordering and linear nonmatch groups.

Unlike the previous experiments, the illustrations drawn by students in the partial ordering condition contained branches intersecting with each other. Even subjects who made errors on their illustrations used intersecting branches in their drawings. This shows that subjects were attempting to represent the comparisons in the form of a partial ordering.

As subjects in the partial ordering condition produced representations of partial orderings and not representations of linear orderings, the data from Experiment 3 could be examined to see whether a serial position learning curve would emerge in the partial ordering condition. the performance of each of the adjacent

Table 4

Mean Proportion of Correct Scores on the Recognition Test for the Three Experimental Conditions in Experiment 3

\begin{tabular}{|c|c|c|c|c|c|c|}
\hline \multirow[b]{2}{*}{ Trial } & \multicolumn{2}{|c|}{ Partial Ordering } & \multicolumn{2}{|c|}{ Linear Nonmatch } & \multicolumn{2}{|c|}{ Linear Match } \\
\hline & Adjacent & Nonadjacent & Adjacent & Nonadjacent & Adjacent & Nonadjacent \\
\hline 1 & .53 & .54 & .57 & .56 & .63 & .61 \\
\hline 2 & .62 & .65 & .60 & .60 & .73 & .79 \\
\hline 3 & .73 & .75 & .72 & .72 & .89 & .92 \\
\hline 4 & .78 & .82 & .80 & .80 & 91 & .91 \\
\hline 5 & .86 & .85 & .84 & .83 & .97 & .97 \\
\hline 6 & .86 & .90 & .87 & .91 & .98 & .97 \\
\hline
\end{tabular}


Table 5

Mean Proportion of Correct Scores on the Illustration Test for the Three Experimental Conditions in Experiment 3

\begin{tabular}{cccc}
\hline Trial & $\begin{array}{c}\text { Partial } \\
\text { Ordering }\end{array}$ & $\begin{array}{c}\text { Linear } \\
\text { Nonmatch }\end{array}$ & $\begin{array}{c}\text { Linear } \\
\text { Match }\end{array}$ \\
\hline 1 & .15 & .18 & .20 \\
2 & .35 & .33 & .54 \\
3 & .51 & .55 & .71 \\
4 & .66 & .65 & .87 \\
5 & .74 & .72 & .98 \\
6 & .81 & .84 & .99 \\
\hline
\end{tabular}

comparisons was calculated across trials; the mean proportions correct for each encoding condition are shown in Table 6. (In this table, Comparison 1 was the first presented in the acquisition phase, Comparison 2 was the second presented, etc.) As can be seen from this table, in the partial ordering condition, there was no evidence of a serial position curve, either down the main axis of the array (Comparisons 1-7), or for the total array (using Comparison 13 as the last end term). Instead, there was a steady linear trend for the first seven pairs of the array, with the first pair receiving the highest score, the next pair the next highest score, and so on, until Pair 8 was reached. Comparisons 8-13 received much lower scores than the first seven comparisons in the array. The last six comparisons form the branches of the partial ordering. Thus, it appears that subjects learned the ordering in a "sequential" fashion, with those comparisons forming the branches of the array being much more difficult to learn than those forming the main axis.

In the linear match condition, there was also evidence of a sequential learning strategy, except that the last couple of comparisons in the array received a somewhat higher score than those in the middle of the array. Thus, there was a slight serial position learning

Table 6

Mean Proportion of Correct Responses for Each Adjacent Pair in Experiment 3

\begin{tabular}{rcccc}
\hline & \multicolumn{4}{c}{ Order } \\
\cline { 2 - 4 } $\begin{array}{c}\text { Adjacent } \\
\text { Pair }\end{array}$ & $\begin{array}{c}\text { Partial } \\
\text { Ordering }\end{array}$ & $\begin{array}{c}\text { Linear } \\
\text { Nonmatch }\end{array}$ & $\begin{array}{c}\text { Linear } \\
\text { Match }\end{array}$ & $\begin{array}{c}\text { Array } \\
\text { Linear } \\
\text { Nonmatch }\end{array}$ \\
\hline 1 & .91 & .90 & .93 & .90 \\
2 & .80 & .81 & .86 & .81 \\
3 & .75 & .75 & .81 & .75 \\
4 & .69 & .65 & .76 & .65 \\
5 & .64 & .57 & .71 & .57 \\
6 & .59 & .49 & .66 & .49 \\
7 & .55 & .41 & .64 & .41 \\
8 & .37 & .39 & .62 & .32 \\
9 & .31 & .41 & .62 & .36 \\
10 & .36 & .45 & .62 & .39 \\
11 & .35 & .32 & .64 & .41 \\
12 & .35 & .36 & .67 & .45 \\
13 & .30 & .66 & .77 & .66 \\
\hline
\end{tabular}

curve in the linear match condition. A similar type of pattern emerged in the linear nonmatch condition when the comparisons were ordered not as they were presented, but as they actually appeared in the array. Thus, in both linear conditions, subjects appeared to learn the earliest presented comparisons first, then the end anchors, and then, finally, the comparisons in the lower middle of the array.

Perfect scores. In the linear match conditions, there were 5 perfect scores on the recognition test on Trial 3 , 6 on Trial 4, 11 on Trial 5, and 10 on Trial 6; on the illustration test, there were 7 perfect scores on Trial 3 , 10 on Trial 4, 17 on Trial 5, and 19 on Trial 6. In the linear nonmatch condition, there was 1 perfect score on the recognition test on Trial 4,6 on Trial 5 , and 7 on Trial 6; on the illustration test, there were 5 perfect scores on Trial 4, 6 on Trial 5, and 13 on Trial 6. In the partial ordering condition, there was 1 perfect score on the recognition test on Trial 4, 4 on Trial 5 , and 6 on Trial 6; on the illustration test, there were 2 perfect scores on Trial 4, 5 on Trial 5, and 10 on Trial 6. This shows that in all conditions there were a number of subjects who learned to correctly represent the arrays.

\section{Discussion}

In Experiment 3, the subjects taught the partial ordering performed as well on the recognition test and on the illustration test as the subjects taught the linear nonmatch ordering. Thus, subjects who had the prototype for a partial ordering in their knowledge systems found a partial ordering as easy to learn as a similarsized linear ordering when that linear ordering contained the same number of nonmatch components as the partial ordering. Complex partial orderings such as the one used in the present experiments must always be presented so that some adjacently presented pairs do not contain a common element. Linear orderings can be presented so that adjacently presented pairs always contain a common element. In Experiment 3, both the partial ordering and the linear nonmatch groups performed significantly more poorly on the two tests than did the linear match group. Thus, when the nonmatch factor was not controlled, the partial ordering was more difficult to learn than the linear ordering.

Experiment 3 also examined the number of correct responses for each comparison pair. This examination suggested that, basically, the subjects in all three encod. ing conditions employed a sequential learning strategy. In other words, the first pair in the acquisition list was the one most often correctly produced, the second pair was the second most often correctly produced, and so on. There were two differences, however, between the strategy employed by subjects in the linear ordering conditions and the strategy employed by subjects in the partial ordering condition. First, in the partial ordering condition, a large difference in correct productions occurred between the last comparison on the main axis 
of the array and the comparisons forming the branches of the partial ordering. This suggests that the comparisons forming these branches were particularly difficult for subjects to learn. In the linear ordering conditions, there was no large break in the sequence. Second, in the linear ordering conditions, the last couple of pairs in the sequence were more often correctly produced than those pairs immediately above them. This suggests that subjects in the linear ordering conditions did find the pairs at both ends easier to learn than the pairs in the middle of the array. In the partial ordering condition, the last couple of pairs were not more often correctly produced than the pairs immediately above them. Thus, in the linear ordering conditions, there was some evidence of a serial position learning curve; in the partial ordering condition, no serial position learning curve emerged.

\section{GENERAL DISCUSSION}

The experiments in this paper were designed to examine whether partial orderings were more difficult to learn than linear orderings of similar size. DeSoto $(1960,1961)$ suggested that partial orderings were more difficult to construct than linear orderings of similar length, but a recent study by Smith and Mynatt (Note 1) found no differences in difficulty of constructing the two types of orderings. The present experiments were designed to investigate this question using longer sequences than were used by Smith and Mynatt.

With longer sequences, it was found that there are two reasons why partial orderings are likely to be more difficult for subjects to acquire than similar-sized linear orderings. First, when subjects are presented a long set of comparative relations, they attempt to arrange the items into a linear array rather than into a partial ordering. They do not appear to possess a general rule specifying that a long set of comparative relations can be represented in other than a simple linear fashion. Probably, this is because they have never before encountered partial orderings of this type. Once they are shown a prototype of a partial ordering, however, they have no difficulty in forming the correct type of representation.

The second reason that complex partial orderings are likely to be more difficult for subjects to learn is that they contain "nonmatch" components. With complex partial orderings, it is impossible for all pairs in the ordering to be presented in a sequential order. No matter how the items are presented, there must be some adjacently presented pairs that do not contain a common element. On the other hand, linear orderings are generally presented in a sequential order. Foos et al. (1976) have shown that it is more difficult for subjects to construct an integrated representation from a nonsequential or "nonmatch" presentation order than from a sequential or "match" presentation order. Thus, it was necessary to rule out this factor in comparing the acquisition of a partial ordering with that of a linear ordering. In Experiment 3, it was found that subjects learned a partial ordering as quickly as they learned a linear ordering that contained the same number of "nonmatch" components, but they performed significantly more poorly than subjects who learned a linear ordering presented in a sequential order. Therefore, it appears that complex partial orderings are not more difficult for subjects to learn than linear orderings of the same length if (1) subjects know how to correctly represent a partial ordering, and (2) both types of orderings have the same number of nonmatch components.

Another question of concern in the present study was whether subjects use a different strategy in learning a partial ordering from that used in learning a linear ordering. With linear orderings, subjects make more errors on the pairs in the middle of the ordering than on those at the ends (e.g., Riley \& Trabasso, 1974; Trabasso et al., 1975). In contrast, Moeser and Tarrant (1977) found no evidence of a serial position learning curve in their investigations of partial orderings. The Moeser and Tarrant study, however, was not designed to look at acquisition processes, and thus it could not offer a definitive answer to this question. The present study was designed specifically to examine acquisition processes in partial orderings, and it also found no evidence of a serial position learning curve in the partial ordering condition. There was no decrease in errors either at the end of the main axis of comparisons or at the end of the series of comparisons. In contrast, in both linear ordering conditions, there was a slight decrease in errors at the end of the array. With both types of orderings, however, subjects performed best on the first comparisons to be presented.

One other variable was examined in these experiments: whether there were any differences in accuracy of judging adjacent and nonadjacent comparative relations. In Experiment 2, performance on adjacent comparison questions was better than performance on nonadjacent comparison questions. This finding replicated one by Moeser and Tarrant (1977). In both studies, performance was better on adjacent comparisons when the adjacent comparisons were well learned and subjects were unable to correctly represent the information they had learned as a partial ordering. In Experiments 1 and 3 , there were no significant differences between adjacent and nonadjacent comparison test questions. This contrasts with the often reported finding that subjects are consistently more accurate in judging nonadjacent relations (e.g., Potts et al., 1978). The superiority in making nonadjacent judgments, however, has only been found on comparative orderings containing a relatively small number of terms. Moeser and Tarrant did not find any differences in accuracy or in response rate between adjacent and nonadjacent comparisons with subjects who correctly learned a 12 -term partial ordering. Woocher (1976), who used an eightitem linear ordering, did not obtain a step-size effect independent of serial position effects ( $\mathrm{cf}$. Woocher, 1975, p. 67). In Experiment 3, a small serial position 
effect was obtained on the two linear orderings. If all possible nonadjacent comparisons had been tested in the recognition measure, performance on the nonadjacent questions might have been slightly superior to performance on the adjacent questions. However, only a limited set of nonadjacent questions was used in the test. Because these questions mainly compared items located in the middle portion of the array, performance on them should have been relatively independent of any serial position effects. Therefore, the fact that the studies in the present article found no significant differences between adjacent and nonadjacent comparisons is consistent with recent findings that the distance effect "is an artifact due to the confounding of step-size with distance from the beginning and either end of the list" (Woocher, 1976, p. 67).

\section{REFERENCE NOTE}

1. Smith, K. H., \& Mynatt, B. T. Effects of presentation order on construction of complete and partial orders. Paper presented at the meeting of the Psychonomic Society, Denver, November 1975.

\section{REFERENCES}

Bowe R, G. H. Adaptation-level coding of stimuli and serial position effects. In M. H. Appley (Ed.), Adaptation-level theory. New York: Academic Press, 1971.

DESoTo, C. B. Learning a social structure. Journal of Abnormal and Social Psychology, 1960, 60, 417-421.

DESoтo, C. B. The predilection for single orderings. Journal of Abnormal and Social Psychology, 1961, 62, 16-23.
DeSoto, C. B., London, M., \& Handel, S. Social reasoning and spatial paralogic. Journal of Personality and Social Psychology, $1965,2,513-521$.

Foos, P. W., Smith, K. H., Sabol, M. A., \& Mynatt, B. T. Constructive processes in simple linear order problems. Journal of Experimental Psychology: Human Learning and Memory, 1976, 2, 759-766.

HAyEs-Roth, B., \& HAYes-Roth, F. Plasticity in memorial networks. Journal of Verbal Learning and Verbal Behavior, 1975, 14, 506-522.

MoESER, S. D. Recognition processes in episodic memory. Canadian Journal of Psychology, 1977, 31, 41-70.

MoESER, S. D. Effect of questions on prose unitization. Journal of Experimental Psychology: Human Learning and Memory, 1978, 4, 290-303.

Moeser, S. D., \& TARrant, B. L. Learning a network of comparisons. Journal of Experimental Psychology: Human Learning and Memory, 1977, 3, 643-659.

Potts, G. R., Banks, W. P., Kosslyn, S. M., Moyer, R. S., RILEY, C. A., \& Smith, K. H. Distance effects in comparative judgments. In N. J. Castellan \& F. Restle (Eds.), Cognitive theory (Vol. 3). Hillsdale, N.J: Erlbaum, 1978.

Riley, C. A., \& Trabasso, T. Comparatives, logical structures, and encoding in a transitive inference task. Journal of Experimental Child Psychology, 1974, 17, 187-203.

Trabasso, T., Riley, C. A., \& Wilson, E. G. The representation of linear order and spatial strategies in reasoning: A developmental study. In R. Falmagne (Ed.), Psychological studies of logic and its development. Hillsdale, N.J: Erlbaum, 1975.

Woochen, F. Storage and retrieval of information from artificial linear orderings. Unpublished doctoral thesis, Stanford University, 1976.

(Received for publication January 22, 1979; revision accepted August 1, 1979.) 